\title{
A Laboratory Simulation for Vectoring of Trichosporon pullulans by Conidia of Botrytis cinerea
}

\author{
Darryl W. M. Cook
}

\begin{abstract}
Sustainable Disease Management Group, The Horticultural and Food Research Institute of New Zealand, Ruakura Research Center, Private Bag 3123, Hamilton, New Zealand. Accepted for publication 24 July 2002.
\end{abstract}

\begin{abstract}
Cook, D. W. M. 2002. A laboratory simulation for vectoring of Trichosporon pullulans by conidia of Botrytis cinerea. Phytopathology 92:1293-1299.

A mechanism that could contribute to the suppression of Botrytis cinerea during pathogen sporulation was examined in this study. Yeasts capable of binding to $B$. cinerea were formulated with a cellulose carrier and applied to sporulating colonies of the pathogen. The particles from this yeast/cellulose product attached to $B$. cinerea conidia in the sporulating colony. Inoculum from treated colonies was harvested and applied

compared with cellulose-only controls. However, between 5 and $11 \%$ of conidia applied were attached to yeast cells. The removal of conidia not attached to yeast resulted in inoculum composed of $>90 \%$ of conidia attached to yeast, and from this inoculum, disease development was significantly retarded $(P<0.05)$. When inoculum from treated $B$. cinerea colonies was applied to nutrient limiting agar and then incubated, the $B$. cinerea conidia germinated, and yeast cells infested the new hyphal growth. Constraints of the formulation of the yeast used in this study, and the implications of this vectoring approach for the suppression of $B$. cinerea during pathogen sporulation are discussed.
\end{abstract} to tomato stem tissue to test for subsequent pathogenicity. Disease development from inoculum obtained from cultures that had been treated with Trichosporon pullulans was significantly retarded $(P=0.0001)$
Additional keywords: adhesion, settling chamber, sporulation, timing of inoculum application, yeast.
The phytopathogen Botrytis cinerea (Pers.:Fr) is an economically important pathogen in many temperate crops (3). Use of fungicides as the principle means of disease management is no longer acceptable because of resistance development within the pathogen population (1) and declining consumer approval (12). The development of biological control strategies is viewed as a suitable substitute for chemical-based technologies.

Biocontrol treatments often require prophylactic application in order to allow establishment of the biocontrol agent (BCA) prior to pathogen arrival (7). However, recent studies have explored the application of antagonists after B. cinerea inoculation. Cook et al. (6), showed that bacteria and yeasts applied $48 \mathrm{~h}$ after $B$. cinerea challenge resulted in significant suppression of infection. Suppression of sporulation as a biocontrol strategy has also been demonstrated in numerous studies $(13,14,17,23)$ where BCA application was made to host tissue with visible lesions caused by $B$. cinerea $(8,18)$, and in some instances the presence of the pathogen could be beneficial for the development of the antagonist. Yeast popultions proliferated following application to $B$. cinerea lesions due to the release of nutrient from the infection process (8). Suppression of sporulation can be exerted following substantial delay periods, for example, Gliocladium roseum Bain. was applied $120 \mathrm{~h}$ after $B$. cinerea challenge (18). However, it was unclear whether sporulation had commenced at this time (18), while in other studies, the onset of sporulation was clearly noted (8). To date, no reports of successful biocontrol following BCA application to sporulating $B$. cinerea were found.

Conidia of $B$. cinerea are borne on determinate highly branched conidiophores that project from the host surface (11). Conidia and conidiophores exposed to the external environment could provide

Corresponding author: D. Cook; E-mail address: cookwurms@clear.net.nz

Publication no. P-2002-1004-01R

(c) 2002 The American Phytopathological Society a suitable surface for attachment by suitable microorganisms. Attachment has been reported for the suppresion of $B$. cinerea infection $(6,7,21)$ and was used as a biocontrol candidate selection criterion (6) from which several antagonists were selected $(5,6)$. However, the application of antagonists, in particular those capable of attaching to the pathogen, to the hyphosphere during pathogen sporulation has not been widely reported, and the effects of BCA colonization of the hyphosphere and to conidia while on the conidiophore, are therefore, poorly understood.

Should attachment by a suitable microbe to conidia during sporulation be achieved, then it is plausible that the attached microbe could be vectored by $B$. cinerea conidia as they are dispersed by natural mechanisms. There are examples in which BCAs are vectored to flowers using honey bees $(16,19)$, and mycoviruses could be considered to be vectored by the pathogen itself (10). In order to demonstrate the potential of this proposed mechanism, it is necessary to identify those microorganisms that can be vectored by $B$. cinerea and show antagonistic traits in this biocontol approach. A collection of yeasts capable of attaching to B. cinerea (6) was examined for this purpose. This paper describes work carried out on the selection of yeasts for the application to sporulating $B$. cinerea using a laboratory simulation of vectoring by liberated pathogen conidia.

\section{MATERIALS AND METHODS}

Preparation of pathogen cultures and formulated yeast. Cultures of B. cinerea isolate BC20, (6) were grown on potato dextrose agar (PDA) (Difco Laboratories, Detroit, MI) for 10 days at $18^{\circ} \mathrm{C}$ and exposed to $12-\mathrm{h}$ photoperiods from near-UV lights (Sankyo FL30 BLB, 30W) and fluorescent lights (NEC 30SSBR$\mathrm{HG}$ ) located $40 \mathrm{~cm}$ above the cultures.

The yeasts, Candida sake (Saito \& Ota) van Uden \& Buckley (isolates 561, 532, and 662dia), C. pulcherrima (Lindner) Windisch (isolate 662dib), Trichosporon pullulans (Lindner) Diddens \& Lodder (isolate 622b), and Galactomyces geotrichum 
(E.E. Butler \& L.J. Peterson) Redhead \& Malloch group A (isolates 552c and 572c) isolated and described previously (6), were stored in $15 \%$ glycerol at $-20^{\circ} \mathrm{C}$ until required. The yeasts were grown in a nutrient yeast dextrose broth (NYDB) composed of $8 \mathrm{~g}$ liter ${ }^{-1}$ nutrient broth (Difco Laboratories), $5 \mathrm{~g}_{\text {liter }}{ }^{-1}$ yeast extract (Difco Laboratories), $10 \mathrm{~g}$ liter $^{-1} \mathrm{D}$-glucose (BDH Laboratory Supplies, Poole, UK) and $\mathrm{pH}$ adjusted to 4.5 with $5 \mathrm{M} \mathrm{HCl}$. A $100-\mathrm{ml}$ volume of NYDB was inoculated with $15 \mu \mathrm{l}$ of each yeast in glycerol then incubated at $18^{\circ} \mathrm{C}$ in 250 -ml baffled conical flasks shaken on an orbital shaker at $150 \mathrm{rpm}$ for $72 \mathrm{~h}$.

The spent culture broth was removed by centrifuging the yeast culture at $766 \times g$ for 5 min (Jouan MR-22 centrifuge, Saint Herblain, France). The yeast cells were resuspended in $30 \mathrm{ml}$ sterile distilled water (SDW). Sigmacell (Sigma S3504, St. Louis, MO) composed of $20-\mu \mathrm{m}$ cellulose particles and added to the yeast suspension at a rate of $1 \mathrm{~g} \mathrm{ml}^{-1}$. The cellulose/yeast suspensions were thoroughly shaken and centrifuged as above to remove the liquid. The cellulose/yeast paste was thinly spread over the base of a sterile glass petri dish (90-mm diameter) and placed under a low vacuum at $25^{\circ} \mathrm{C}$ for $16 \mathrm{~h}$ to dry. Each cellulose/yeast preparation was then powdered using a coffee grinder (Breville Model CG2, Breville Holdings Pity Ltd., Australia) set at maximum speed for $30 \mathrm{~s}$.

Comparison of yeast isolates. A laboratory procedure was developed to simulate each of the critical processes that were likely to occur in the vectoring of yeast by liberated $B$. cinerea conidia. The cellulose/yeast formulation was applied to sporulating $B$. cinerea cultures grown on PDA using a settling chamber (4). Plastic transfer pipets (Corning Samco Corp., San Fernando, CA) were modified by removing most of the barrel, but retaining the bulb plus a $1 \mathrm{~cm}$ long portion of the barrel with $0.4 \mathrm{~g}$ of the dry yeast powder loaded into the bulb portion of the pipet. The yeast/ cellulose powder was discharged into the settling chamber by puncturing a small hole in the side of the bulb and injecting a short $(<1 \mathrm{~s})$ pulse of compressed $\mathrm{CO}_{2}$ (400 $\mathrm{kPa}$ gauge pressure) into the bulb (4). The yeast particles were allowed to settle during a 4 min period on the sporulating $B$. cinerea cultures situated on the settling chamber base. A control treatment composed of Sigmacell alone was applied to $B$. cinerea.

Inoculum liberation from a $B$. cinerea colony was simulated by removing and collecting inoculum comprising of $B$. cinerea conidia and conidia attached to particles from the yeast/cellulose product or attached to Sigmacell-only using a Cyclone surface sampler (Burkard Manufacturing, Rickmansworth, UK). The inoculum was thoroughly mixed. To simulate deposition, aliquots

TABLE 1. Disease development ( $\log _{10}$ area under disease progress curve) from Botrytis cinerea conidia vectoring yeasts

\begin{tabular}{lcccc}
\hline & \multicolumn{4}{c}{ Dose $^{\mathrm{y}}$} \\
\cline { 2 - 5 } Treatment $^{\mathrm{z}}$ & $1.0 \mathrm{mg}$ & $5.0 \mathrm{mg}$ & $10.0 \mathrm{mg}$ & $20.0 \mathrm{mg}$ \\
\hline Cellulose & $1.00 \mathrm{a}$ & $1.24 \mathrm{a}$ & $1.42 \mathrm{a}$ & $1.50 \mathrm{a}$ \\
561 & $0.81 \mathrm{~b}$ & $1.18 \mathrm{a}$ & $1.37 \mathrm{a}$ & $1.43 \mathrm{~b}$ \\
532 & $1.01 \mathrm{a}$ & $1.32 \mathrm{a}$ & $1.40 \mathrm{a}$ & $1.48 \mathrm{a}$ \\
$662 \mathrm{dia}$ & $1.00 \mathrm{a}$ & $1.23 \mathrm{a}$ & $1.40 \mathrm{a}$ & $1.43 \mathrm{~b}$ \\
$662 \mathrm{dib}$ & $0.95 \mathrm{a}$ & $1.25 \mathrm{a}$ & $1.37 \mathrm{a}$ & $1.40 \mathrm{~b}$ \\
$552 \mathrm{c}$ & $0.98 \mathrm{a}$ & $1.12 \mathrm{~b}$ & $1.37 \mathrm{a}$ & $1.41 \mathrm{~b}$ \\
$572 \mathrm{c}$ & $1.00 \mathrm{a}$ & $1.18 \mathrm{a}$ & $1.36 \mathrm{a}$ & $1.38 \mathrm{~b}$ \\
$622 \mathrm{~b}$ & $0.67 \mathrm{~b}$ & $0.77 \mathrm{~b}$ & $1.19 \mathrm{~b}$ & $1.33 \mathrm{~b}$ \\
\hline
\end{tabular}

$\mathrm{y}$ The vectored yeast was applied to sporulating Botrytis cinerea colonies prior to the collection of conidia. Aliquots of 1.0, 5.0, 10.0, and $20.0 \mathrm{mg}$ of this inoculum composed of $B$. cinerea conidia and yeast were prepared, and applied to the tomato stem pieces using the settling chamber. Common letters within each column signify that the associated treatment does not significantly differ from the cellulose control according linear contrasts $(P>0.05)$. Probability values for those treatments that are significantly different are given in the text.

${ }^{\mathrm{z}}$ The treatments included Sigmacell alone (cellulose), Candida sake (isolates 561, 532, and 662dia), C. pulcherrima (isolate 662dib), T. pullulans (isolate $622 \mathrm{~b}$ ), and Galactomyces geotrichum (isolates 552c and 572c) formulated in Sigmacell cellulose. of $1.0,5.0,10.0$, or $20.0 \mathrm{mg}$ of the inoculum from each treatment or control were prepared in eppendorf tubes and applied to tomato stem pieces and two water agar (WA) coated microscope slides using the settling chamber (4). Stem pieces from tomato plant ( $L y$ copersicon esculentum cv. MoneyMaker) seedlings were prepared as previously described where tomato plants were grown in a bark-based medium for 7 weeks, harvested, and leaves and petioles removed (6). Each stem was cut into $15 \mathrm{~mm}$ long pieces and held in a "cassette" (6) for inoculation of the wound area and the incubation period of 10 days at $18^{\circ} \mathrm{C}$. Five replicate cassettes each containing 10 stem pieces were prepared. Each stem piece was inspected, and successful infection was recorded when $B$. cinerea sporulation became visible under $\times 10$ magnification. Assessments of pathogen sporulation were carried out at 24-h intervals, and the proportion of those stems exhibiting pathogen sporulation was recorded. These values were integrated over the time period of the observations to calculate the area under the disease progress curves (AUDPC). The experiment was repeated once.

Activity of the yeast T. pullulans. The isolate of T. pullulans (622b) was selected for further study due to its superior disease suppression abilities. The following treatments were prepared: (i) Yeast $622 \mathrm{~b}$, as described above; (ii) boiled $622 \mathrm{~b}$ was prepared by boiling the $622 \mathrm{~b}$ cells for $15 \mathrm{~min}$ prior to formulating with Sigmacell; (iii) Sigmacell-only; and (iv) boiled Sigmacell-only controls were composed of the cellulose product and prepared in the same manner as for the yeast treatments just described. Each yeast/cellulose treatment or Sigmacell control was applied to $B$. cinerea colonies at concentrations of $0.2,0.4$, or $0.6 \mathrm{~g}$ using the settling chamber. The inoculum that was harvested from these treated colonies was prepared as 1.0, 5.0, and $10.0 \mathrm{mg}$ doses and applied to 20 tomato stem pieces ( 10 stem pieces per cassette) and to two WA coated microscope slides using the settling chamber (4). Each inoculum dose was applied to tomato stem pieces in three replicates.

The composition and density of the inoculum from each of the four treatments and inoculum doses was analyzed by examining the WA coated microscope slides at $\times 160$ magnification using a Leitz Dialux microscope set to dark field. Spore densities were calculated for three categories of conidia: (i) Unattached $=$ the number of $B$. cinerea spores that were not attached to particles from either the yeast/cellulose product or the Sigmacell control; (ii) attached minus $(-) 622 \mathrm{~b}=$ the number of visible $B$. cinerea spores attached to Sigmacell-only or to the yeast/cellulose product with nonviable $622 \mathrm{~b}$ cells. There was no budding in the nonviable yeast; and (iii) attached plus (+) $622 \mathrm{~b}=$ the number of visible $B$. cinerea spores attached to particles from the yeast/cellulose product with viable $622 \mathrm{~b}$ cells. The area of observation was defined by the diameter of the field of view $(1 \mathrm{~mm})$ and the distance (in $\mathrm{mm}$ ) traversed along the slide during the counting procedure.

Since only a small percentage of the inoculum applied using the settling chamber was composed of conidia attached to particles from the cellulose/yeast, it was necessary to eliminate unattached B. cinerea conidia from the inoculum applied in the tomato stem bioassay. Using an alternative inoculum application method, $5.0 \mathrm{mg}$ of inoculum harvested from treated sporulating $B$. cinerea colonies was released into the top of a $2.8 \mathrm{~m}$ high, $0.1 \mathrm{~m} \times 0.1 \mathrm{~m}$ vertical column constructed from carton grade cardboard. Inoculum from the same four treatments just described was applied. The faster rate of descent by the conidia attached to yeast particles compared with the unattached conidia allowed for a substantial separation of the two inoculum types. Beneath the column outlet, two cassettes, each with 10 tomato stem pieces and two WA coated microscope slides were positioned and remained exposed to the column output for a period of 2 min immediately following inoculum release. Fresh cassettes and WA coated microscope slides were exposed to the column output during a second 2-min period. The experiment was repeated eight times. 
Scanning electron microscope studies. The WA coated slides used in the analysis of the composition and density of inoculum applied in the $T$. pullulans experiments above were retained and used for scanning electron micrography (SEM) image analysis. One of the slides from each treatment was immediately prepared for SEM while the other was incubated at $4^{\circ} \mathrm{C}$ for 10 days. Microscope slides coated with PDA were also prepared, and inoculum from the same treatments was applied using the column method of inoculation. All of the PDA coated slides were incubated at $4^{\circ} \mathrm{C}$ for 10 days.

Five replicate specimens together with the thin layer of agar were lifted from the glass slide and were exposed to vapors from a $2 \%$ solution of osmium tetroxide (ProSciTech, Thuringowa, Qld,
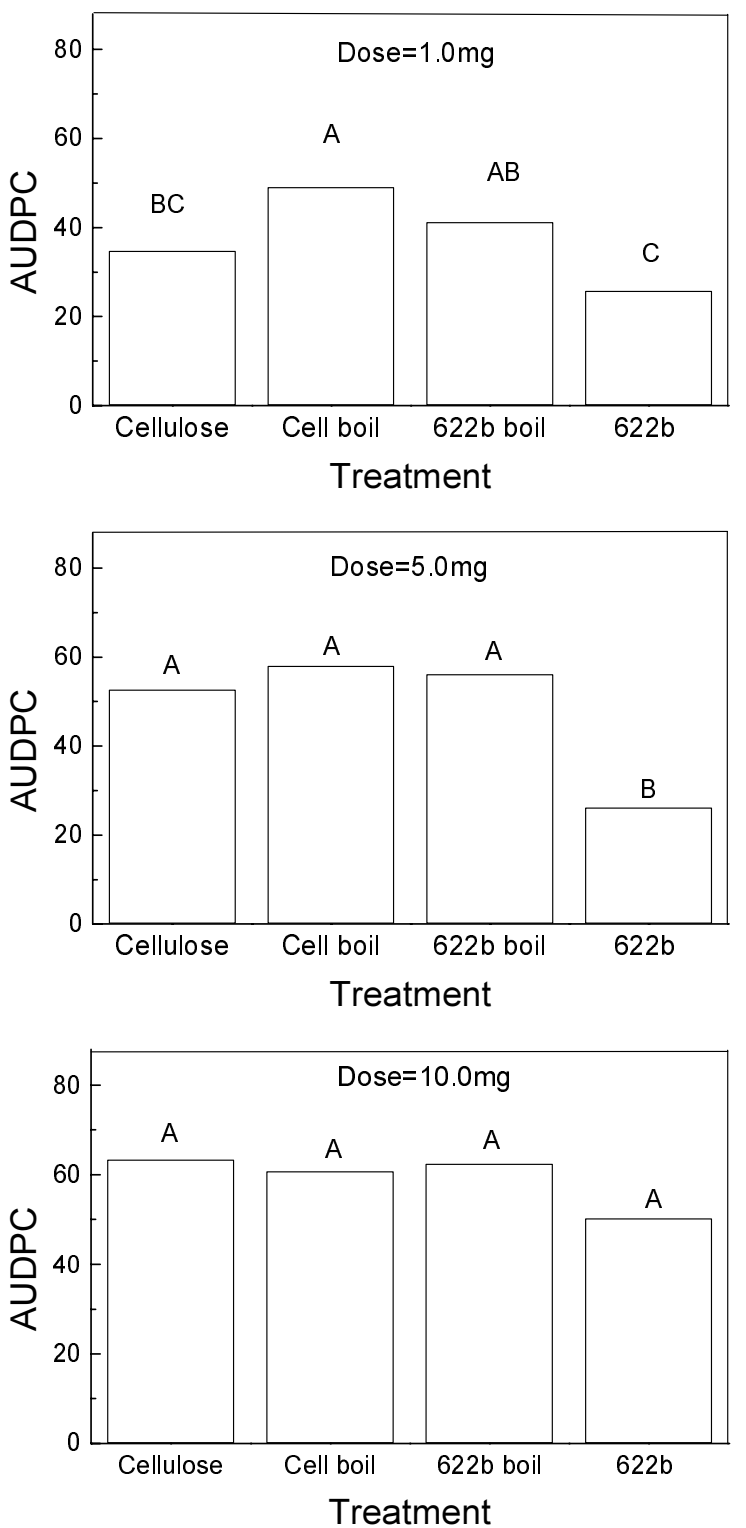

Fig. 1. Disease development in tomato stem tissue from Botrytis cinerea conidia vectoring the yeast Trichosporon pullulans (isolate 622b). Treatments were as follows: Cellulose $=$ Sigmacell only, Cell boil $=$ Sigmacell boiled, $662 \mathrm{~b}$ boil $=T$. pullulans formulated with Sigmacell (isolate 622b) boiled, and $622 \mathrm{~b}=$ viable $T$. pullulans formulated with Sigmacell. Each treatment was applied to sporulating $B$. cinerea colonies prior to the collection of conidia. Aliquots of 1.0, 5.0, and $10.0 \mathrm{mg}$ were prepared and applied to the tomato stem pieces. Treatments were replicated three times. AUDPC $=$ Area under the disease progress curve. Letters in common indicate treatments that are not significantly different $(P>0.05)$ according to Tukey's analysis.
Australia) (for a period of $16 \mathrm{~h}$ at room temperature). Following a $16-\mathrm{h}$ venting period, the specimens were dehydrated in a sealed petri dish filled with silica crystals for $24 \mathrm{~h}$ at room temperature. The dried samples were platinum/palladium coated using a Hitachi E1030 Ion sputter coater and viewed using a Hitachi, S4000 Field Emission SEM. Images were recorded using digital image capture equipment. The experiment was conducted once with SEM and three times with light microscopy.

Statistical analysis. Data from all experiments conducted in this work was analyzed using analysis of variance (ANOVA) and The SAS System (version 6.12, SAS Institute, Cary, NC). For those experiments examining pathogen sporulation in the tomato stem pieces, AUDPC was the response variable used in ANOVA.
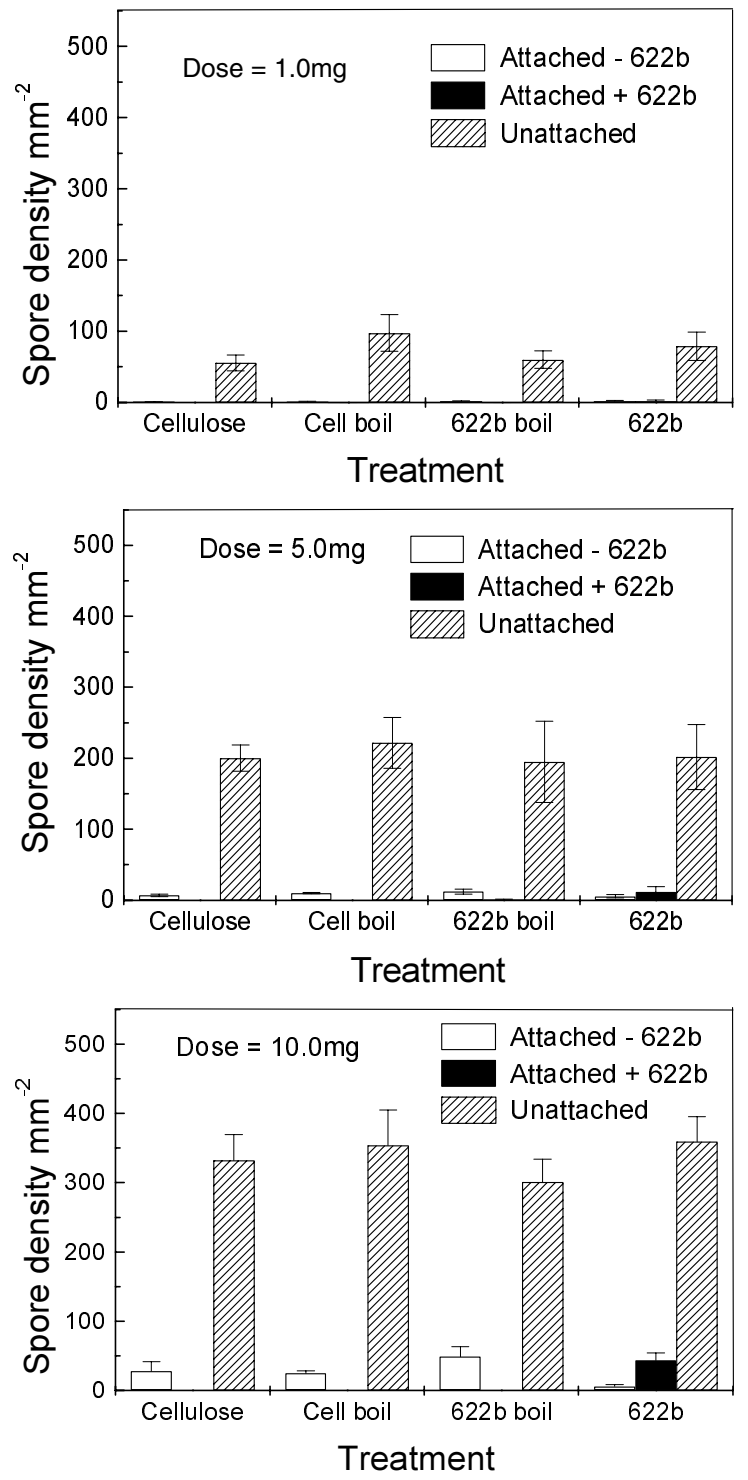

Fig. 2. Spore density of inoculum obtained from sporulating Botrytis cinerea colonies treated with the yeast Trichosporon pullulans and then applied to tomato stem pieces and water-agar coated microscope slides. The attachment of $B$. cinerea spores to particles from the treatments was defined as: (i) Unattached $=$ the number of $B$. cinerea spores that were not attached to particles from the yeast/cellulose product; (ii) Attached minus (-) $622 \mathrm{~b}=$ the number of visible $B$. cinerea spores attached to cellulose only or $B$. cinerea conidia attached to cellulose with nonviable $622 \mathrm{~b}$ cells. There was no budding in the nonviable yeast; and (iii) Attached plus (+) $622 \mathrm{~b}=$ the number of visible $B$. cinerea spores attached to particles from the yeast/cellulose product with viable $622 \mathrm{~b}$ cells. The inoculum was applied at 1.0, 5.0, and $10.0 \mathrm{mg}$ using a settling chamber. Error bars are standard errors of means from three replicates. 
For the comparison of yeast isolates, the data was $\log _{10}$ transformed in order to comply with assumptions of ANOVA, and significant treatment effects were distinguished using linear contrasts $(P<0.05)$, in which each yeast treatment effect was compared with the Sigmacell control. The various treatments included in examining the activity of $T$. pullulans were compared using Tukey's multiple range analysis with a critical probability of $P=0.05$.

\section{RESULTS}

Comparison of yeast isolates. Disease development in tissue inoculated with treated $B$. cinerea inoculum was significantly retarded compared with disease progress in control treatments (Table 1). At all of the doses applied, inoculum obtained from $B$. cinerea cultures treated with $T$. pullulans (isolate $622 \mathrm{~b}$ ) resulted in significantly $(P=0.0009$ to 0.0001$)$ reduced disease development. There were also significant reductions in disease development for C. sake (isolate 561) in $1.0 \mathrm{mg}$ of inoculum $(P=0.03)$ and for $G$. geotrichum (isolate 552c) in $5.0 \mathrm{mg}$ of inoculum $(P=0.03)$. When $20.0 \mathrm{mg}$ of inoculum was applied, there were significant reductions in disease development by $C$. sake (isolates $561[P=0.02]$ and 662dia $[P=0.01]$ ), $C$. pulcherrima (isolate 662dib $[P=$ $0.002]$ ) and $G$. geotrichum (isolates $552 \mathrm{c}[P=0.007]$ and $572 \mathrm{c}$ $[P=0.0003])$.

Activity of the yeast $T$. pullulans. The effect of the different concentrations $(0.2,0.3$, or $0.4 \mathrm{~g})$ of the yeast $T$. pullulans (isolate $622 \mathrm{~b}$ ) applied to the sporulating $B$. cinerea on subsequent disease
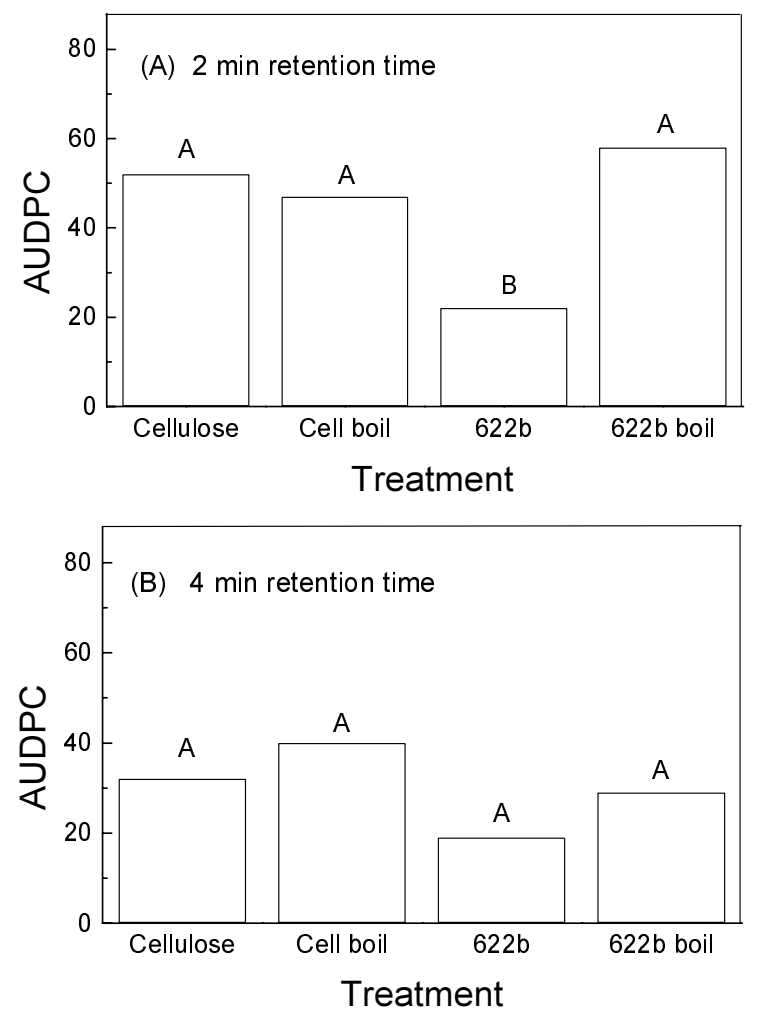

Fig. 3. Disease development in tomato stem tissue resulting from Botrytis cinerea conidia vectoring the yeast Trichosporon pullulans (isolate 622b). Inoculum was applied with a $2.8 \mathrm{~m}$ high column where: $\mathbf{A}$, = particles emerged from the column during the first 2-min retention period after inoculation and $\mathbf{B},=$ particles emerging after a second 2-min retention period. Treatments were: Cellulose $=$ Sigmacell only, Cell boil $=$ Sigmacell boiled, and $662 \mathrm{~b}$ boil $=T$. pullulans (isolate $622 \mathrm{~b}$ ) boiled. Each treatment was applied to sporulating $B$. cinerea colonies prior to the collection of conidia, aliquots of $5.0 \mathrm{mg}$ were prepared and applied to the tomato stem pieces using the $2.8 \mathrm{~m}$ column. Treatments were replicated eight times. AUDPC $=$ Area under the disease progress curve. progress was not significant $(P=0.82$; data not shown). Therefore, the data were pooled according to treatment and inoculum dose and is presented in Figure 1. The presence of the yeast isolate $622 \mathrm{~b}$ in pathogen inoculum significantly retarded disease progress $(P<0.05)$ compared with inoculum containing boiled cellulose-only or boiled $622 \mathrm{~b}$ at a dose of $1.0 \mathrm{mg}$. There was no significant difference $(P>0.05)$ between $622 \mathrm{~b}$ treatment and cellulose-only control. In $5.0 \mathrm{mg}$ of inoculum, disease progress was significantly retarded $(P<0.05)$ with conidia from $622 \mathrm{~b}-$ treated $B$. cinerea colonies compared with all controls. At the same dose, there was no significant difference in AUDPC among all three controls $(P>0.05)$. At the highest dose, $10.0 \mathrm{mg}$, there were no significant differences $(P>0.05)$ among any of the treatments or controls (Fig. 1).

The density of conidia increased as larger inoculum doses were applied to the tomato stem pieces (Fig. 2). In the 1.0, 5.0, and $10.0 \mathrm{mg}$ dose, the average conidial density was 50 spores $\mathrm{mm}^{-2}$, 200 spores $\mathrm{mm}^{-2}$, and 350 spores $\mathrm{mm}^{-2}$, respectively (Fig. 2). In all treatments and doses, the inoculum applied was dominated by conidia that were not attached to BCA particles (Fig. 2).

Disease development in tomato tissue inoculated using the $2.8 \mathrm{~m}$ high column, and from the inoculum that had emerged during the first 2 -min period was significantly $(P<0.05)$ retarded compared with the control treatments (Fig. 3A). Inoculum that emerged during this period was dominated by conidia attached to particles with no viable $622 \mathrm{~b}$ cells in the cellulose-only, boiled cellulose-only, boiled $622 \mathrm{~b}$ treatment, or viable $622 \mathrm{~b}$ cells in the $622 \mathrm{~b}$ treatment (Fig. 4A). Overall, the quantity of inoculum that
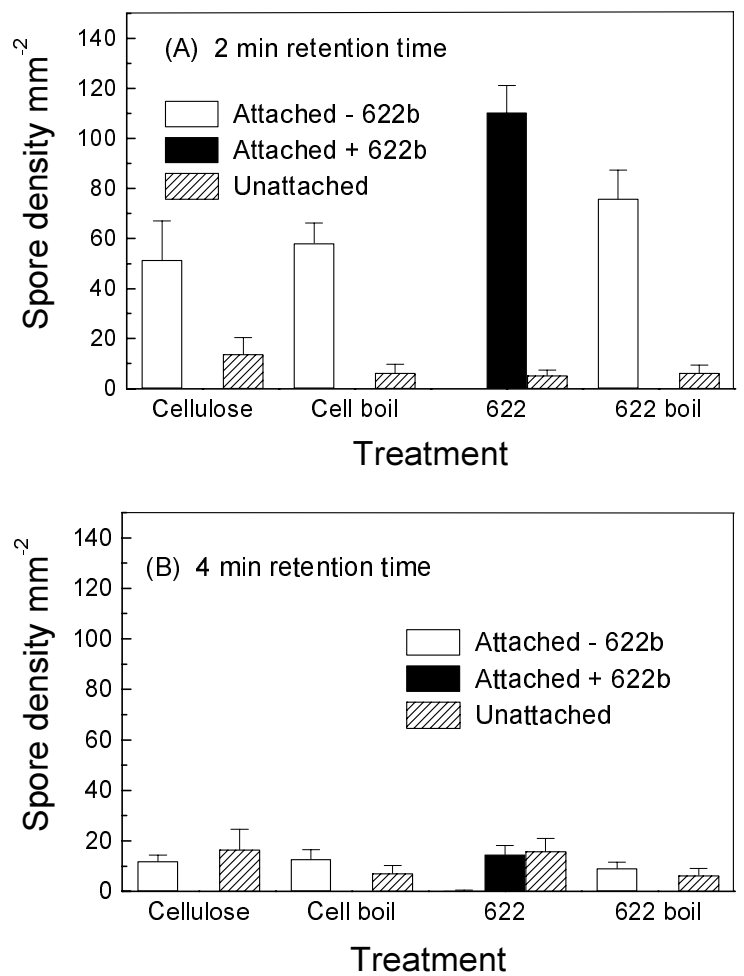

Fig. 4. Spore density of inoculum obtained from sporulating Botrytis cinerea colonies treated with the yeast Trichosporon pullulans that emerged from a $2.8 \mathrm{~m}$ high column during $\mathbf{A}$, the first 2 -min retention period after inoculation and $\mathbf{B}$, after a second 2-min retention period (See Materials and Methods). The attachment of $B$. cinerea spores to particles from the treatments was defined as: (i) Unattached = the number of $B$. cinerea spores that were not attached to particles from the yeast/cellulose product; (ii) Attached minus $(-) 622 \mathrm{~b}=$ the number of visible $B$. cinerea spores attached to cellulose only or $B$. cinerea conidia attached to cellulose with nonviable $622 \mathrm{~b}$ cells. There was no budding in the nonviable yeast; and (iii) Attached plus $(+) 622 \mathrm{~b}=$ the number of visible $B$. cinerea spores attached to particles from the yeast/cellulose product with viable $622 \mathrm{~b}$ cells. 
emerged from the column during the second 2-min period (Fig. 4B) was considerably lower compared with the first period and no particular category of attachment classification dominated. In the corresponding disease bioassay (Fig. 3B), there were no significant treatment differences $(P>0.05)$.

Scanning electron microscope studies. The yeast, $T$. pullulans formulated with Sigmacell, produced a fine powder that was composed of particles approximately $80 \mu \mathrm{m}$ in size (data not shown). SEM investigation of the inoculum obtained from $622 \mathrm{~b}$-treated sporulating $B$. cinerea colonies showed many $B$. cinerea conidia surrounding the cellulose/yeast particles (Fig. 5). These $B$. cinerea conidia appeared to be attached to yeast cells that were coating the exterior of the cellulose particle (Fig. 6). After incubation on nutrient limiting media (water agar), B. cinerea conidia had germinated producing hyphae (Fig. 5) and the hyphae were infested with yeast cells from 622b (Figs. 5 and 7) many of which were concentrated about the hyphal tip (Fig. 7). In contrast, inoculum obtained from $622 \mathrm{~b}$-treated sporulating $B$. cinerea colonies and applied to PDA, showed considerable pathogen and yeast growth but no infestation of hyphal growth by the yeast (Fig. 8).

\section{DISCUSSION}

An isolate of the yeast T. pullulans was identified by the laboratory simulation as a potentially effective antagonistic microbe that could be vectored by conidia of $B$. cinerea and could also retard disease development. Under the incubation conditions imposed in this study, T. pullulans was unique among the yeasts tested because the isolate was able to multiply and grow on $B$. cinerea hyphae during pathogen germination.

A number of recent studies have examined the biocontrol opportunities with application of BCA after B. cinerea establishment for the suppression of infection (6) or spore production $(8,17,18)$. A range of time periods between $B$. cinerea application and BCA application have been described, but due to differences in host and inoculation methods, the extent of pathogen development at the time of BCA application will be different, and therefore, it is difficult to compare these studies. Nevertheless, a common feature is the BCA application during the pathogen latent period in which disease symptoms were visible but sporulation

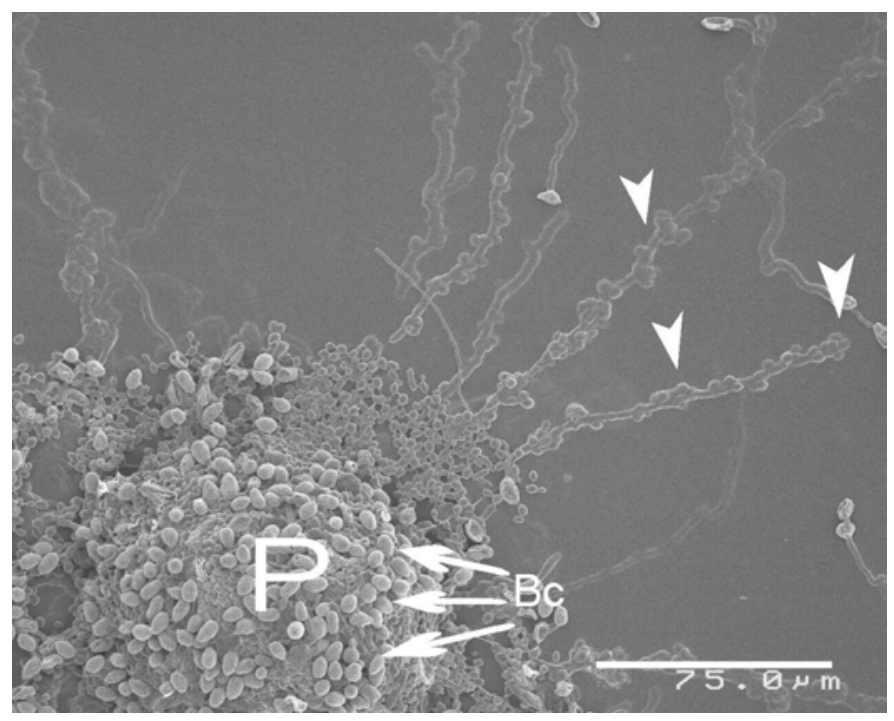

Fig. 5. Scanning electron microscopic image of inoculum obtained from Botrytis cinerea sporulating colony that was treated with the yeast Trichosporon pullulans. The method of formulating the yeast resulted in yeast/cellulose particles $(\mathrm{P})$ to which $B$. cinerea conidia $(\mathrm{Bc})$ attached to the exterior. The treated $B$. cinerea inoculum was applied to water-agar and incubated at $4^{\circ} \mathrm{C}$ whereupon $B$. cinerea conidia germinated (arrowheads) and the hyphal surface was infested with cells from $T$. pullulans (arrowheads). had not commenced. BCA application to sporulating B. cinerea colonies presents a number of biocontrol challenges including the suppression of the conidia that are already present in the sporulating lesion. Furthermore, at sporulation, pathogen establishment has progressed to an extent that the resources such as nutrient and space have been acquired to produce conidia. Thus, the numerous mechanisms already described for the suppression of $B$. cinerea (7) are unlikely to be effective for these reasons. To address these issues, an alternative biocontrol strategy was explored in the present study, in which $B$. cinerea conidia were exploited to carry the potential to the next site of infection. During the proceeding infection process the vectored BCA is activated and suppression of infection takes place.

Vectoring of BCAs for the timely dispersal of agents to flowers has been previously reported $(16,19)$. However, the use of the pathogen itself as the vector and that the vectoring mechanism

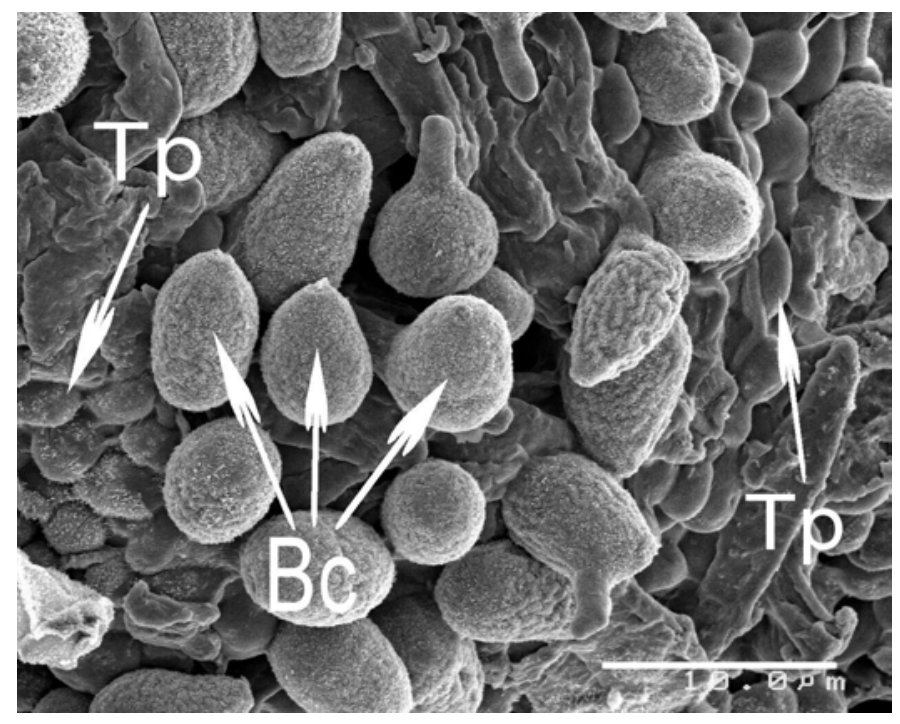

Fig. 6. Scanning electron microscopic image of inoculum obtained from Botrytis cinerea sporulating colony that was treated with the yeast Trichosporon pullulans. The image is showing a higher magnification view of the surface of the particle shown in Figure 5, covered in the yeast cells from $T$. pullulans $(\mathrm{Tp})$ and $B$. cinerea conidia $(\mathrm{Bc})$. Note that several $B$. cinerea conidia have germinated.

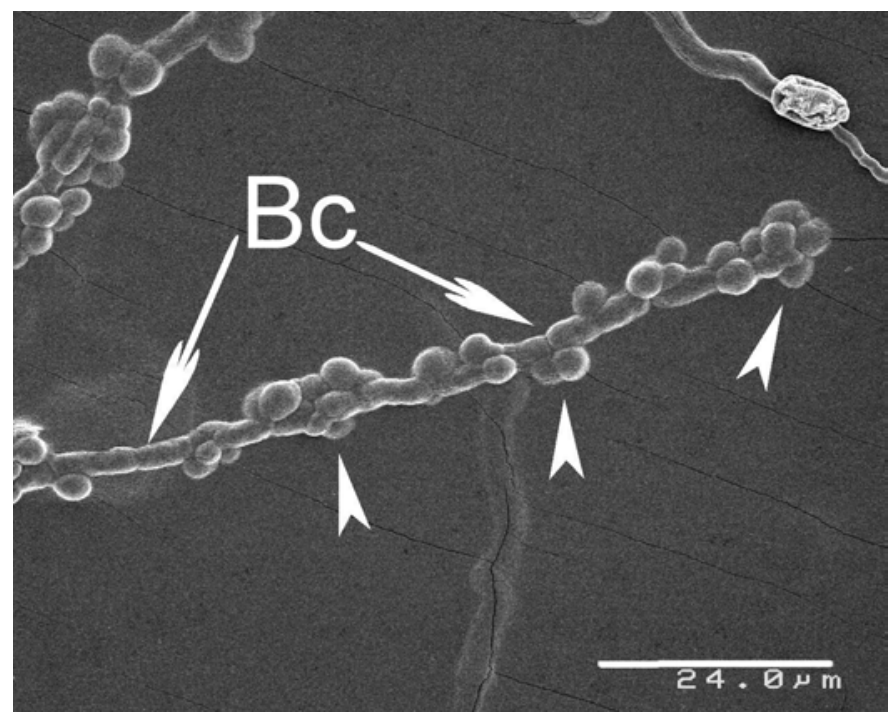

Fig. 7. Scanning electron microscopic image of a Botrytis cinerea hypha (Bc) that had grown from the particles shown in Figure 5, infested by many cells of the yeast Trichosporon pullulans and in particular about the terminal region of the hypha (arrowheads). 
will require spray application to the host plant as the primary method of introducing the BCA into the environment, distinguish this approach from previous vectoring studies. A bioassay for vectored biocontrol was used in the laboratory in which conidia were harvested from treated sporulating colonies and applied to tomato stem tissue. This procedure allowed for accurate doses of the various treatments and controls to be applied, in order to compare treatments, and to establish a dose-pathogenicity relationship so that effective yeast agents could be identified.

The yeast was formulated with the Sigmacell because of the requirements to preserve the viability of the yeast and the attachment competence by the yeasts to structures in sporulating $B$. cinerea colonies. In previous studies, the same yeasts were applied as cells suspended in water to the host tissue in a bioassay to determine the suppression of B. cinerea infection (6). However, the application of these yeasts suspended in water to sporulating pathogen colonies did not result in strong yeast attachment, whereas applying freeze dried yeast cells resulted in yeast attachment to conidiophores and conidia of B. cinerea (D. Cook, unpublished data). The application of the dry yeast/cellulose product to $B$. cinerea colonies resulted in a bond between $B$. cinerea conidia and particles from the yeast/cellulose product that appeared to be strong enough to withstand the sheer forces in the cyclone within the vacuum surface sampler. In the present study, the addition of the cellulose prior to drying the yeast cells significantly enhanced cell viability during the drying of the product. The Sigmacell product used for this purpose has an average particle size of $20 \mu \mathrm{m}$ (Sigma). However, the preparation with the yeast cells resulted in a powder composed of particles of such a size that facilitated the adhesion of many $B$. cinerea conidia to the exterior of a single particle as shown in Figures 2 and 4 and SEM images (Fig. 6). The particles from the yeast/cellulose product could in fact be considered the carrier of the pathogen. Clearly, this is a departure from the original vectoring model in which the antagonist, such as the yeast cell, is transported by a larger host such as $B$. cinerea conidia. However, an essential element of vectoring, attachment of the two microbes, and the simultaneous transportation of both to a new infection site was achieved and this resulted in retarded disease development.

The composition of inoculum obtained from treated sporulating $B$. cinerea colonies, and inoculum applied using the settling chamber was dominated by pathogen conidia not attached to

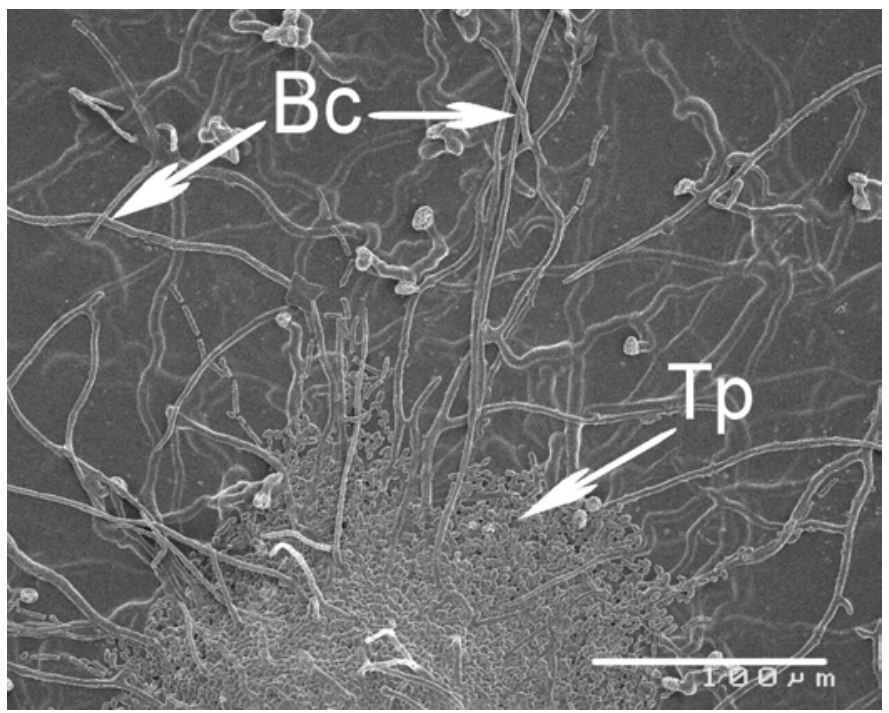

Fig. 8. Scanning electron microscopic image of inoculum obtained from Botrytis cinerea sporulating colony that was treated with the yeast Trichosporon pullulans applied to potato dextrose agar and incubated at $4{ }^{\circ} \mathrm{C}$. Note the abundant growth of $B$. cinerea $(\mathrm{Bc})$ and the yeast $T$. pullulans $(\mathrm{Tp})$ but there is no infestation of the hyphal surface by the yeast. either cellulose particles or to yeast/cellulose particles from the formulated product. The presence of these conidia in the inoculum had the potential to confound any biocontrol affects that could be due to the vectored antagonist. The unattached conidia were removed using the $2.8 \mathrm{~m}$ high column because as the large cellulose particles had a considerably faster rate of descent, they could be separated during free-fall from unattached single conidia. In the subsequent bioassay, in which the tomato stem pieces were exposed only to the attached conidia, the biocontrol effects could be more accurately ascribed to $T$. pullulans attached to $B$. cinerea. However, other potentially confounding influence created by the numerous $B$. cinerea conidia attached to the particles remained.

Infection aggression by $B$. cinerea was correlated with the number of conidia per droplet applied to several host plants $(15,20)$. Coertze and Holz (2) proposed that differences in host response to different inoculation methods were due to the application of dispersed individual conidia compared with groups of conidia in water droplets. Highly clumped distributions of conidia led to a collapse in host resistance due to increased inoculum pressure. In the present study, the high concentration of pathogen conidia surrounding each yeast particle could have increased disease pressure placing high demands on the biocontrol efficacy of $T$. pullulans. Total suppression of disease from inoculum obtained from yeasttreated colonies was not observed in this study, yet the application of $T$. pullulans as a suspension resulted in complete suppression of $B$. cinerea infection in the same tomato stem bioassay (6). Furthermore, the pathogen inoculum that was composed of between 5 and $11 \%$ of conidia that were attached to yeast particles (Fig. 2) retarded disease development by $56 \%$ compared with the control (Fig. 1). Inoculum applied using the chromatographic approach was composed of $95 \%$ of conidia attached to yeast (Fig. 4), and retarded disease development by $58 \%$ compared with the control (Fig. 3). The reason for this disproportionately small improvement in the level of biocontrol despite the removal of unattached conidia, remains unknown. It is possible that the ratio of the number of $B$. cinerea conidia attached to each yeast/cellulose particle, perhaps determined by the contact made between product and the sporulating pathogen, constrained the ability of the yeast to suppress $B$. cinerea. Different rates of the yeast/cellulose product were applied to sporulating $B$. cinerea colonies in an attempt to alter the amount of contact between the pathogen conidia and the yeast product and influence biocontrol efficacy. Since applying different rates of the yeast/cellulose product did not result in a significant effect, suggests that other unknown factors require investigation.

During the incubation of treated inoculum on nutrient limiting media, T. pullulans cells (isolate $622 \mathrm{~b}$ ) consistently infested the surface of $B$. cinerea hyphae (Figs. 5 and 7). There was no hyphal infestation by 622 b when $B$. cinerea was grown on PDA (Fig. 8), or by any other yeast isolate tested in the present study (data not shown). Further work will be required to further elucidate the reasons for this infestation phenomenon. Among a number of possible hypotheses could include a nutritional dependence by the yeasts on $B$. cinerea due to the superior competitive ability of the pathogen. The idea of an effective BCA with inferior competitive ability that may result in pathogen suppression is contrary to conventional hypotheses (7). Competition has been implicated as Yang et al. (22) showed that in the absence of pyoverdine production in a mutant strain of Pseudomonas sp., biocontrol activity was lost despite retaining attachment competence. Alternatively, changes in the composition of the pathogen extra cellular matrix with different media could prevent yeast adhesion of this $T$. pullulans isolate or the secretion from the pathogen hyphae of an exudate that is beneficial for yeast growth cannot be excluded.

Fokkema (9) stated that the entire life cycle of the pathogen should be examined for opportunities for biocontrol. In the present study, the emergence of an isolate of T. pullulans as an antagonist, demonstrates that yeasts can be isolated and used in the vectoring 
manner described in the present study to suppress pathogen activity during $B$. cinerea sporulation. To date, biocontrol strategies have targeted pathogen infection or spore production with BCAs applied as a prophylactic treatment or after $B$. cinerea establishment but prior to pathogen sporulation $(13,14,18)$. Following successful suppression during sporulation, it is now possible to consider that the entire life cycle of $B$. cinerea could be suppressed by microbial antagonist activity. Therefore, biocontrol as a disease management tool may no longer be restricted to preventative treatments. With the use of yeasts such as $T$. pullulans employed as a vectored BCA, applications made during advanced phases of epidemic development could arrest further epidemic development and limit future crop losses caused by $B$. cinerea diseases. However, many aspects of this work require further study including the epidemiological implications of this vectored biocontrol strategy in a cropping situation.

\section{ACKNOWLEDGMENTS}

The author wishes to thank the New Zealand Foundation for Research, Science, and Technology for providing a FRST Postdoctoral Fellowship to fund this work; and T. Reglinski and K. V. Wurms for valuable comments regarding the manuscript.

\section{LITERATURE CITED}

1. Beever, R. E., Laracy, E. P., and Pak, H. A. 1989. Strains of Botrytis cinerea resistant to dicarboximide and benzimidazole fungicides in New Zealand vineyards. Plant Pathol. 38:427-437.

2. Coertze, S., and Holz, G. 1999. Surface colonization, penetration, and lesion formation on grapes inoculated fresh or after cold storage with single airborne conidia of Botrytis cinerea. Plant Dis. 83:917-924.

3. Coley-Smith, J. R., Verhoeff, K., and Jarvis, W. R. 1980. The Biology of Botrytis. Academic Press, London.

4. Cook, D. W. M., Elmer, P. A. G., and Hill, R. A. 2002. Quantitative inoculation and infection of tomato stems with dry conidia from Botrytis cinerea. Australas. Plant Pathol. 31:217-222.

5. Cook, D. W. M., Long, P. G., and Ganesh, S. 1999. The combined effect of delayed application of yeast biocontrol agents and fruit curing for the inhibition of the postharvest pathogen Botrytis cinerea in kiwifruit Postharv. Biol. Technol. 16:233-243.

6. Cook, D. W. M., Long, P. G., Ganesh, S., and Cheah, L.-H. 1997. Attachment microbes antagonistic against Botrytis cinerea-biological control and scanning electron microscope studies in vivo. Ann. Appl. Biol. 131: 503-518.

7. Elad, Y. 1996. Mechanisms involved in the biological control of Botrytis cinerea incited diseases. Eur. J. Plant Pathol. 102:719-732.

8. Elad, Y., Kohl, J., and Fokkema, N. J. 1994. Control of infection and sporulation of Botrytis cinerea on bean and tomato by saprophytic yeasts. Phytopathology 84:1193-1200.

9. Fokkema, N. J. 1995. Biological control of foliar fungal diseases. Pages 67-176 in: Biological Control: Benefits and Risks. H. M. T. Hokkanen and J. M. Lynch, eds. Cambridge University Press, Cambridge.

10. Howitt, R. L. J., Beever, R. E., Pearson, M. N., and Forster, R. L. S. 1995. Presence of double-stranded RNA and virus-like particles in Botrytis cinerea. Mycol. Res. 99:1472-1478.

11. Jarvis, W. R. 1977. Botryotinia and Botrytis species: Taxonomy, physiology, and pathogenicity. Monogr. 15. Can. Dep. Agric. Res. Branch, Ottawa.

12. Klingauf, F. A. J. 1995. Registration requirements of biological control agents in Germany and in the European Union. Pages 283-290 in: Biological Control: Benefits and Risks. H. M. T. Hokkanen and J. M. Lynch, eds. Cambridge University Press, Cambridge.

13. Kohl, J., Molhoek, W. M. L., van der Plas, C. H., and Fokkema, N. J. 1995. Suppression of sporulation of Botrytis spp. as a valid biocontrol strategy. Eur. J. Plant Pathol. 101:251-259.

14. Kohl, J., Molhoek, W. M. L., van der Plas, C. H., and Fokkema, N. J. 1995. Effect of Ulocladium atrum and other antagonists on sporulation of Botrytis cinerea on dead lily leaves exposed to field conditions. Phytopathology 85:393-401.

15. Mansfield. J. W. 1980. Mechanisms of resistance to Botrytis. Pages 181218 in: The Biology of Botrytis. J. R. Coley-Smith, K. Verhoeff and W. R. Jarvis, eds. Academic Press, London.

16. Peng, G., Sutton, J. C., and Kevan, P. G. 1992. Effectiveness of honey bees for applying the biocontrol agent Gliocladium roseum to strawberry flowers to suppress Botrytis cinerea. Can. J. Plant Pathol. 14:117-129.

17. Sutton, J. C., and Peng, G. 1993. Biocontrol of Botrytis cinerea in strawberry leaves. Phytopathology 83:615-621.

18. Szandala, E. S., and Backhouse, D. 2001. Suppression of sporulation of Botrytis cinerea by antagonists applied after infection. Australas. Plant Pathol. 30:165-170.

19. Vanneste, J. L., Cornish, D. A., Voyl, M. D., Haine, H. M., and Goodwin, R. M. 1999. Honey bees to distribute beneficial bacteria to apple and Asian pear flowers. Proc. Eighth Int. Workshop on Fire Blight. Acta Hortic. 489:615-617.

20. Verhoeff, K. 1980. The infection process and host-pathogen interactions. Pages 153-180 in: The Biology of Botrytis. J. R. Coley-Smith, K. Verhoeff, and W. R. Jarvis, eds. Academic Press, London.

21. Wisniewski, M., Biles, C., Droby, S., McLauglin, R., Wilson, C. L., and Chalutz, E. 1991. Mode of action of the postharvest biocontrol yeast, Pichia guilliermondii 1. Characterisation of attachment to Botrytis cinerea. Physiol. Mol. Plant Pathol. 39:259-267.

22. Yang, C-H., Menge, J. A., and Cooksey, D. A. 1994. Mutations affecting hyphal colonization and pyoverdine production in Pseudomonads antagonistic toward Phytopthora parasitica. Appl. Environ. Microbiol. 60: 473-481.

23. Zhang, P. G., Sutton J. C., and Hopkin, A. A. 1996. Inoculum concentration and time of application of Gliocladium roseum in relation to biocontrol of Botrytis cinerea in black spruce seedlings. Can. J. For. Res. 26:360-367. 